\title{
O NÍVEL DE SERVIÇO DO TECON RIO GRANDE PELA ÓTICA DOS EXPORTADORES E IMPORTADORES
}

\section{THE SERVICE LEVEL OF TECON RIO GRANDE BY EXPORTERS AND IMPORTERS PERCEPTION}

\author{
Guilherme Bergmann Borges Vieira ${ }^{1}$; Roberto Birch Gonçalves ${ }^{2}$; Giovana Savitri Pasa ${ }^{3}$; Francisco \\ José Kliemann Neto ${ }^{4}$ \\ ${ }^{1}$ Universidade de Caxias do Sul- UCS - RS - Brasil \\ gbvieira@cpovo.net \\ ${ }^{2}$ Universidade de Caxias do Sul- UCS - RS - Brasil \\ rbgoncal@ucs.br \\ ${ }^{3}$ Universidade Federal do Rio Grande do Sul - UFRGS - RS - Brasil \\ giovanapasa@producao.ufrgs.br \\ ${ }^{4}$ Universidade Federal do Rio Grande do Sul - UFRGS - RS - Brasil \\ kliemann@producao.ufrgs.br
}

\begin{abstract}
Resumo
A competitividade das empresas no contexto internacional depende, entre outros fatores, do aprimoramento de seu processo logístico como um todo. E tal processo está fortemente relacionado ao modal marítimo, uma vez que esse modal representa cerca de $90 \%$ dos embarques internacionais de carga. Os portos, por sua vez, funcionam como elos da cadeia logística global, afetando tanto os custos como o nível de serviço logístico e, conseqüentemente, influenciando a competitividade dos setores exportadores e importadores que deles se utilizam. Nesse contexto, o nível de serviço portuário merece especial atenção, sendo necessário avaliá-lo constantemente, com vistas a seu aprimoramento. O presente artigo teve como objetivo identificar e avaliar os atributos competitivos do Tecon Rio Grande a partir da ótica dos exportadores e importadores da serra gaúcha, usuários de seus serviços. $O$ nível de serviço do Tecon em cada atributo, comparativamente ao nível de serviço dos portos concorrentes, foi avaliado pelos próprios respondentes e a importância de cada atributo foi obtida de forma indireta, por meio de análise de regressão múltipla. O estudo gerou subsídios tanto para a melhoria dos serviços por parte do terminal quanto para a (re) avaliação da escolha portuária por parte dos usuários, uma vez que identificou a importância dos distintos fatores considerados pelos usuários na escolha portuária e avaliou o desempenho do Tecon nos mesmos.
\end{abstract}

Palavras-chave: nível de serviço portuário; exportadores e importadores da serra gaúcha; Tecon Tio Grande. 


\section{Introdução}

Como conseqüência do processo de globalização, as relações comerciais internacionais tem se intensificado. Segundo a Organização Mundial do Comércio (OMC, 2010), o crescimento das exportações mundiais no período de 1950 a 2009 foi, em média, 1,7 vezes superior ao crescimento econômico. Esse índice apresentou um ápice nos anos noventa, havendo um arrefecimento de 2000 a 2010, especialmente devido à redução da taxa de crescimento das exportações, as quais cresceram $3,2 \%$, número consideravelmente inferior à média de 5,8\% observada em todo o período (MILAN; VIEIRA, 2011).

Segundo dados da Organização Mundial do Comércio (OMC, 2011), em 2010 as exportações mundiais de mercadorias aumentaram em 14 por cento em termos de volume, chegando ao valor de US\$ 3.600 bilhões, enquanto que o PIB mundial cresceu 3,5 por cento. Os Estados Unidos se mantiveram como os maiores negociadores mundiais, seguidos pela China e pela Alemanha, respectivamente. Embora o ano de 2009 tenha sido marcado por uma redução de volume e valor no comércio internacional, em 2010 os números se recuperaram. Em termos de movimentação portuária, o Anuário Estatístico Portuário (ANTAQ, 2009), que engloba a movimentação de 119 instalações portuárias, sendo 36 portos organizados e 83 terminais de uso privativo, aponta que a movimentação total de cargas nas instalações portuárias do Brasil em 2009 foi de 732.931.141 toneladas. Além disso, os terminais apresentaram uma movimentação de 473.104.617 t, representando 64,55\% do total movimentado pelo país. Essa porcentagem relativa cresceu nos últimos dois anos, devido ao aumento da quantidade de Terminais de Uso Privativo (TUPs), aos altos investimentos privados e também ao crescimento da produtividade e da eficiência operacional.

Em decorrência da globalização, o papel dos portos tem mudado. Hoje, cada vez mais, os portos devem ser tão "orientados comercialmente" quanto os setores de transporte e comércio exterior que eles servem. Devem funcionar como um centro de serviços aos clientes para que possam garantir a retenção dos mesmos, buscando chegar a portos de $4^{\mathrm{a}}$ geração (PAIXÃO; MARLOW, 2003), os quais passam a se integrar à rede de transportes mundial e buscam a complementaridade em vez da pura e simples competição (SOUSA JUNIOR, 2010).

Um porto lida, basicamente, com dois tipos de clientes: os donos das cargas (embarcadores) e os donos dos navios (armadores). Do ponto de vista dos armadores, a escolha de um porto depende de fatores como: localização geográfica; potencial gerador de cargas; conexões intermodais; disponibilidade e eficiência dos serviços portuários; tarifas praticadas e sistemas de informação. Já, do ponto de vista dos embarcadores (exportadores, importadores, ou agentes de carga prestando serviços para os primeiros) são considerados fatores como: disponibilidade de 
serviços em linha regular (abrangência e cobertura); distância do porto aos pontos de origem/destino das cargas; tarifas portuárias; disponibilidade e eficiência dos serviços, entre outros.

O porto, enquanto importante elo da cadeia de distribuição física internacional (DFI), deve fornecer "valor" a seus clientes, tanto armadores quanto embarcadores, com o objetivo de retenção e fidelização dos mesmos.

O Rio Grande do Sul é um estado com vocação exportadora e o Terminal de Contêineres do Porto de Rio Grande (Tecon Rio Grande) é uma importante alternativa para o escoamento das cargas exportáveis. Essas cargas provêm de diferentes regiões do Estado, tendo especial destaque a região da serra gaúcha. Essa região tem forte vocação industrial, destacando-se os setores metal mecânico e automotivo, o setor de material de transporte, o setor moveleiro, além das indústrias alimentícias e de bebidas, entre outras.

Segundo o Atlas Socioeconômico do Rio Grande do Sul (2010), o setor de veículos automotores, reboques e carrocerias é bastante concentrado espacialmente no Estado. Cinco municípios concentram 52,3\% do total dos estabelecimentos, com destaque para Caxias do Sul, com 25\% do total. Além disso, a indústria de móveis tem na Serra Gaúcha seu principal pólo, sendo que, entre os dez municípios com maior número de estabelecimentos, somente dois não se localizam na Serra Gaúcha. Nos setor de metalúrgica, por sua vez, Caxias do Sul também aparece como o município com maior número de estabelecimentos, destacando-se quanto ao número de empregos gerados. No setor de bebidas também se destacam alguns municípios da serra: quanto ao número de estabelecimentos, o município de Flores da Cunha aparece com 18,2\%, seguido por Caxias do Sul com $14 \%$, Bento Gonçalves com $12 \%$ e Garibaldi com 8,6\% do total de estabelecimentos do setor.

Segundo Bello, Teruchkin e Garcia (2010), no período de 2001 a 2008, as exportações do Rio Grande do Sul sempre representaram um percentual acima de 16\% do PIB estadual, o que demonstra sua importância para a economia do Estado. Além disso, a região da serra destaca-se como uma importante origem das exportações gaúchas. Segundo dados apresentados pelo diretor Comercial do Tecon Rio Grande, Thierry Rios, em palestra na Câmara de Indústria, Comércio e Serviços de Caxias do Sul (CIC) no dia 24/05/2010, a serra gaúcha representa 23\% da movimentação de cargas gerada pelo terminal. No entanto, deve-se considerar a existência de uma considerável concorrência interportuária, especialmente com os portos catarinenses (SANTOS et al, 2007). Segundo os autores, a região serrana do Rio Grande do Sul conta hoje com duas principais alternativas portuárias: o porto de Rio Grande e o porto de Itajaí, em Santa Catarina. No entanto, para cargas consolidadas ou LCL (VIEIRA; RODRIGUES, 2005), pode-se considerar também o porto de Santos como uma alternativa viável, devido ao maior número de serviços de linha regular que oferece para esse tipo de carga. Para ilustrar essa problemática, a Figura 1 apresenta a 
localização da região em estudo, bem como a localização dos portos de Rio Grande, Itajaí/ Navegantes, Itapoá e Santos.

A distância entre a região serrana e os Portos de Rio Grande e Itajaí é similar: 440 km e 470 km, respectivamente. Já a distância ao porto de Itapoá, próximo à Joinville, é de aproximadamente $625 \mathrm{~km}$. Até o porto de Santos, a distância é consideravelmente maior $(980 \mathrm{~km})$. No entanto, o tempo associado a tal distância poderia ser compensado, por exemplo, por uma maior frequencia de navios e os custos do transporte rodoviário até Santos poderiam ser compensados pela redução de estoques possibilitada por essa maior frequencia, pelo maior número de armadores que oferecem serviços de linha regular ou pela redução dos fretes em função do maior nível de concorrência existente nesse porto.

Portanto, embora aparentemente as distâncias entre a região em estudo e os distintos portos mencionados possam ser consideradas o principal aspecto na escolha portuária, é importante considerar que tais distâncias podem ser compensadas por benefícios que tornem um porto mais atraente que outro, tanto na questão dos serviços oferecidos como na dos custos logísticos. Trata-se da intersecção entre vários hinterlands (zonas de influência portuária) mencionada por Peyrelongue (1999).

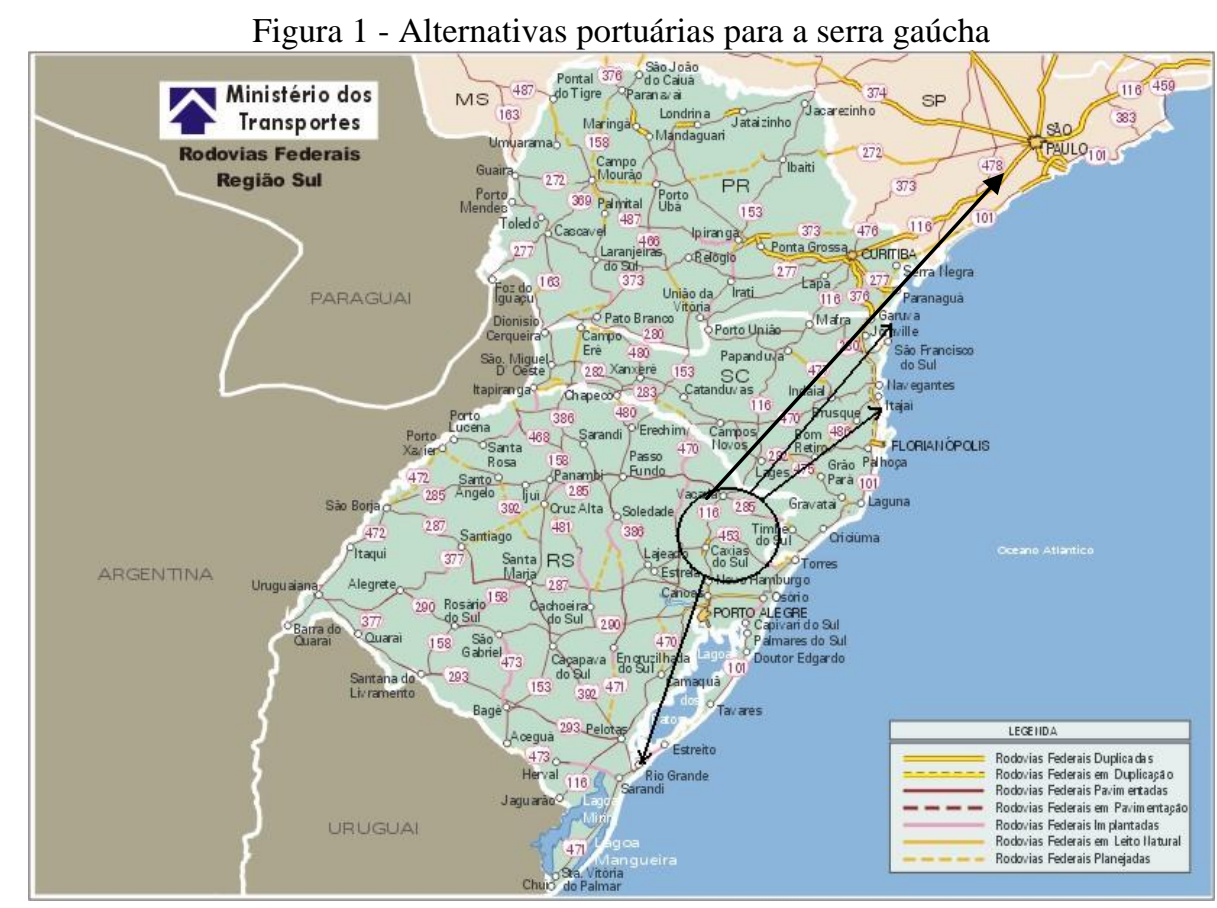

Fonte: Adaptado de Ministério dos Transportes (2006)

Dado o contexto anteriormente descrito e considerado o acirramento da concorrência interportuária e seu impacto no terminal em estudo, para que o mesmo possa gerar satisfação e conseguir a retenção de seus clientes, deve formular uma proposta diferenciada de valor aos mesmos. Essa proposta deve levar em consideração os atributos mais valorizados no momento da escolha portuária e o seu desempenho em cada atributo, comparativamente à concorrência. Diante do exposto, o objetivo geral do presente estudo foi identificar e avaliar os fatores competitivos que 
compõem a proposição de valor do Tecon Rio Grande, a partir da percepção de um grupo de usuários dos seus serviços (empresas exportadoras e importadoras da serra gaúcha). Para tanto, foram estabelecidos os seguintes objetivos específicos: i) identificar os atributos mais importantes na escolha de um terminal de contêineres; ii) avaliar, a partir da percepção dos usuários da amostra, o desempenho do Tecon Rio Grande, comparativamente ao desempenho de seus concorrentes, nos principais atributos de escolha portuária identificados; iii) sugerir ações de melhoria no sentido de proporcionar uma proposta de valor mais aderente às necessidades dos usuários.

A importância do presente estudo justifica-se, primeiramente, por uma maior aproximação e entendimento do setor portuário, enfatizando seu papel na economia global, sua evolução e as novas exigências e desafios a que estarão sujeitos os terminais de contêineres que pretendam manter-se competitivos em um mercado cambiante, concentrado e com elevada concorrência. Adicionalmente, possibilita ao Tecon Rio Grande conhecer a avaliação dada por seus clientes a cada atributo portuário, gerando subsídios para a melhoria dos serviços. E, finalmente, oferece aos usuários uma análise do nível de serviço logístico-portuário no Tecon, apoiando o processo de escolha portuária nas operações de exportação e importação.

\section{Revisão bibliográfica}

A crescente concorrência entre terminais de contêineres, determinada pelo transporte intermodal e pela concentração das rotas marítimas (reduções dos números de escala das companhias proprietárias de embarcações de grande porte), tem ocasionado a mudança da área de influência tradicional de muitos portos, que devem prestar cada vez mais atenção a questões mercadológicas; tal fato justifica o valor do marketing portuário (AGÓS, 1993).

Segundo Arruda e Bastos (2000), a concentração das atividades marítimo-portuárias é um reflexo das tendências de concentração da atividade econômica no contexto da globalização. Sendo assim, o gestor portuário deve atuar como um praticante de marketing, no sentido de identificar consumidores potenciais e mantê-los satisfeitos.

A visão moderna de um porto enquanto empresa condiciona sua gestão às necessidades do cliente. Assim, fica evidente que um porto eficiente, e operacionalmente eficaz, cuida não só de estabelecer tarifas justas e competitivas, mas responde também à ótica dos clientes, para os quais o que realmente interessa é o custo e a eficiência da operação portuária como um todo (ARRUDA; BASTOS, 2000). Esse contexto justifica a avaliação do nível de serviço logístico-portuário e há vários anos esse tema tem sido foco de diversos estudos, contemplando desde uma perspectiva mais ampla do serviço logístico ao cliente até o nível de serviço portuário propriamente dito.

Especificamente na área de transportes, já foram desenvolvidos vários estudos sobre os níveis de serviço e os atributos considerados pelos usuários quando da escolha por um fornecedor. 
Segundo Fleury (2002), os critérios mais utilizados para a avaliação e seleção de um provedor de serviços de transporte, em ordem de importância, são: confiabilidade, preço, flexibilidade operacional e comercial, saúde financeira, qualidade do pessoal operacional e informações de desempenho. Para o autor, a confiabilidade tende a ser o principal critério qualificador - condição mínima para uma empresa ser selecionada - e o preço tende a ser o critério classificador - aquele que irá definir, entre os fornecedores pré-qualificados, qual será o transportador escolhido.

Em termos de transporte marítimo internacional, alguns autores especificam com maior profundidade o que é, pela ótica dos usuários, nível de serviço e quais são os atributos considerados quando da contratação de um transportador. Devido aos avanços no gerenciamento da cadeia de suprimentos, cada vez mais empresas embarcadoras buscam parceiros com maior sofisticação em seus serviços de transporte (KUMAR, 2003). Com esse propósito, o autor recomenda às empresas que avaliem anualmente seus provedores de transporte marítimo internacional sob os seguintes aspectos: i) pontualidade nas entregas; ii) valor dos fretes; iii) tecnologia de informação; iv) serviço ao cliente; e v) equipamentos e operações.

Segundo Tuna e Silan (2002), conhecer os critérios de escolha de usuários de transporte marítimo internacional também é fundamental para a competitividade dos fornecedores desses serviços no mercado atual. Gentry (1996) e Panayides (1998) afirmam que, devido ao desenvolvimento do marketing de relacionamento como um novo paradigma mercadológico, a natureza do relacionamento entre compradores e transportadores tem sofrido significativas mudanças nos últimos anos (apud TUNA; SILAN, 2002). Entende-se marketing de relacionamento como o processo contínuo de identificação e criação de valor para os clientes, criando uma parceria que gerará benefícios duradouros para ambas as partes (GORDON, 1998). Nesse sentido, o relacionamento entre embarcadores e transportadores tem migrado de uma relação de transação compra-e-venda simplesmente - para uma relação estratégica ou de parceria, com vistas a benefícios contínuos de longo prazo (TUNA; SILAN, 2002).

Segundo D’Este e Meyrick (1992), na escolha de um transportador marítimo podem ser considerados tanto fatores qualitativos como quantitativos. Athawale, Mukherjee e Chakraborty (2009), em seu estudo sobre seleção de fornecedores, corroboram essa afirmativa e argumentam que esse tipo de seleção é tipicamente uma decisão multicritério.

Embora sejam encontrados na literatura muitos estudos sobre os critérios de seleção de transportadores marítimos, tais estudos não apresentam um consenso sobre quantos e quais atributos devem ser considerados, havendo tanto proposições detalhadas, com até 40 critérios de avaliação (LU; MARLOW, 1999), como sucintas, com sete ou oito critérios (BROOKS, 1999).

Segundo Brooks (1995), o custo do serviço e a capacidade de resolver problemas são os atributos mais valorizados pelos clientes no processo de escolha de um armador. Já Kent e Parker 
(1999) destacam que confiabilidade, disponibilidade de equipamentos e frequencia de navios são os principais atributos considerados pelos clientes quando da escolha de um transportador marítimo.

Tuna e Silan (2002) realizaram um estudo sobre os critérios de seleção de transportadores de linha regular no Porto turco de Izmir. Trata-se de uma referência interessante por ser um porto com porte similar ao de Rio Grande (em número de contêineres movimentados). Entre os 24 atributos pesquisados, os dez aspectos mais importantes foram: i) entregar a carga sem danos; ii) emitir documentação de embarque corretamente; iii) entregar a carga no prazo acordado; iv) possuir agilidade em lidar com problemas; v) informar mudança de programação de navios; vi) enviar cotações de fretes de forma precisa; vii) responder com rapidez a reclamações; viii) emitir documentação de embarque rapidamente; ix) enviar prontamente remessas urgentes; e x) informar clara e corretamente o cliente sobre custos.

Vieira e Rodrigues (2005), por sua vez, identificaram os principais critérios de escolha de um consolidador de cargas marítimas, tendo como base o estudo de uma empresa desse tipo atuante no porto de Rio Grande. Os autores consideraram como variáveis independentes dez atributos: i) frete; ii) tempo de trânsito; iii) regularidade; iv) frequencia; v) abrangência/cobertura dos serviços; vi) qualidade e agilidade na impressão de documentação de embarque; vii) serviço de informação; viii) atendimento e cordialidade dos colaboradores; ix) preparo técnico da equipe de colaboradores; e x) imagem no mercado/reputação. Além desses atributos, foi considerada também a satisfação geral em relação aos serviços da empresa (variável dependente). No modelo encontrado pelos autores, as variáveis preparo técnico da equipe, agilidade e qualidade na emissão da documentação de embarque, frequencia e regularidade mostraram-se significativas a um nível de confiança de pelo menos $99 \%$ e, utilizando-se tais variáveis, obteve-se um coeficiente $\mathrm{R}^{2}$ de $94,41 \%$, o que indica uma relação relativamente forte entre elas e a satisfação geral.

A partir da análise da literatura da área, também são identificados vários procedimentos para avaliar o nível de serviço logístico e escolher fornecedores desse tipo de serviço. Em sua maioria, os autores sugerem a abordagem perceptiva como uma valiosa ferramenta para a avaliação do nível de serviço logístico. Segundo essa abordagem, as avaliações podem ser classificadas em dois grupos (DALEY; LAMBERT, 1980; D’ESTE, 1992; BAKER, 1984): i) compensatórias e ii) não compensatórias. A abordagem não compensatória parte do pressuposto de que o tomador de decisão não faz uma comparação direta entre os critérios de avaliação, ou seja, a performance em um critério não está relacionada com o desempenho nos demais. Assim, a boa performance em um determinado critério não compensa um desempenho inferior em outro. Por outro lado, a abordagem compensatória analisa todos os atributos em conjunto. Dessa forma, o desempenho em um critério pode aumentar ou reduzir a avaliação geral do nível de serviço de uma empresa. Por exemplo, um desempenho superior em tempo de trânsito ou em flexibilidade pode compensar um custo mais alto. 
No que se refere especificamente ao nível de serviço e aos critérios de escolha portuária, o número de trabalhos encontrados na literatura é mais reduzido, podendo-se citar os estudos de Murphy, Daley e Dalemberg (1992), Murphy e Daley (1994); Novaes e Vieira (1996); Monteiro, Martin e Rodrigues (2001); Tongzon (2002), Vieira e Pasa (2005); Vieira et al (2006); Santos et al (2007), entre outros. A partir de uma análise geral de tais estudos, podem-se identificar os principais critérios de escolha portuária pela ótica dos armadores e pela ótica dos usuários (exportadores, importadores e agentes de carga).

Pela ótica dos armadores, destacam-se critérios como potencial gerador de carga; localização do porto e distância em relação às principais rotas de navegação (main services); capacidade de receber navios de grande porte; considerando-se especialmente o calado; eficiência nas operações de carga e descarga; tempos de espera em fundeio e tarifas portuárias (VIEIRA; PASA, 2005; TRAN, 2011). Percebe-se que esses critérios estão associados aos conceitos de hinterland, vorland e umland, termos oriundos do idioma alemão e amplamente utilizados no jargão portuário. O hinterland diz respeito à área “do porto para trás”, ou seja, o potencial gerador de cargas da área de influência portuária (MUÑOZ, 1989). Já o vorland (do porto para frente) refere-se à área de influência marítima, ou seja, a maior ou menor proximidade do porto às principais rotas de navegação (MUÑOZ, 1989). E o umland, segundo Valpuesta e Manzano (2001), compreende a zona de influência imediata do porto (recinto portuário) e a zona de influência primária (área em que é determinante a presença do porto e cujo desenvolvimento depende em grande medida das atividades portuárias). Portanto, refere-se ao porto em si e à área que o circunda, suas adjacências, compreendendo elementos que conformam a infraestrutura existente e que tem impacto direto no nível de serviço logístico-portuário (eficiência e eficácia dos serviços, tarifas cobradas etc).

Já pela ótica dos usuários, destacam-se fatores como custos portuários, fretes terrestres até o porto ou do porto ao local de destino; número e abrangência de serviços em linha regular; fretes marítimos; frequencia de navios; tempos de trânsito marítimo e porta a porta (TONGZON, 2009; TRAN, 2011).

No que se refere às técnicas aplicadas, destacam-se procedimentos de análise multivariada de dados, tais como regressão múltipla, análise conjunta e análise fatorial (HAIR JR. et al, 2005; MALHOTRA, 2001), estudos multicritério baseados em processo de análise hierárquica - Analythic Hierarchy Process (AHP), seguindo a lógica desenvolvida por Saaty (1991) ou variações da mesma, e aplicações da lógica Fuzzy (MAGALA; SAMMONS, 2008).

\section{Procedimentos metodológicos}

Com base nos procedimentos técnicos utilizados, esta pesquisa pode ser classificada como de levantamento. De acordo com Gil (2002), a pesquisa de levantamento caracteriza-se pela 
interrogação direta das pessoas cuja opinião e atitude se deseja conhecer. Neste caso, foram aplicados questionários às pessoas responsáveis pela escolha portuária nas empresas pertencentes à amostra. Os questionários foram aplicados pessoalmente, durante quatro semanas, no mês de setembro de 2011.

A população da pesquisa correspondeu às empresas exportadoras e importadoras associadas à Câmara de Indústria e Comércio da Caxias do Sul (CIC). Tomando-se como base essas empresas, a amostra foi definida por conveniência e acessibilidade, tomando-se o cuidado de que as empresas selecionadas tivessem experiência de pelo menos três anos no comércio exterior e de que os respondentes fossem os profissionais efetivamente responsáveis pela escolha portuária na empresa.

Os atributos que compuseram o questionário foram os seguintes: i) fretes rodoviários até o porto (FRT); ii) custos portuários (CPO); iii) fretes marítimos (FRM); iv) frequencia de navios (FRQ); v) tempo de trânsito porta a porta (TPO) e vi) atendimento no porto (ATE). Além desses atributos, foi avaliado desempenho geral (GER) do Tecon comparativamente aos concorrentes. A definição desses atributos seguiu a orientação de Novaes e Vieira (1996), Vieira et al (2006) e Santos et al (2007). Foram considerados tais estudos por terem sido desenvolvidos no Brasil, de forma metódica, e por refletirem significativamente as recomendações encontradas na literatura da área.

O instrumento de coleta de dados foi composto por questões para avaliar, pela ótica das empresas pertencentes à amostra, o desempenho do Tecon Rio Grande em cada atributo da lista e, também, para avaliar seu desempenho geral comparativamente aos concorrentes. Além disso, foram incluídas perguntas para qualificar a amostra, identificando aspectos como: setor de atuação das empresas, predomínio das operações (exportação ou importação), número de funcionários (porte); tempo de experiência com exportação e importação; principais destinos das exportações e origens das importações; critérios de escolha portuária considerados mais importantes e cargo de quem respondeu o questionário.

Para a avaliação do desempenho do Tecon Rio Grande em cada atributo, foi utilizada uma escala Likert de cinco pontos (SAMPIERI; COLLADO, LUCIO, 2006), conforme legenda a seguir: (1) Muito pior do que os concorrentes; (2) Pior do que os concorrentes; (3) Igual aos concorrentes; (4) Melhor do que os concorrentes; e (5) Muito melhor do que os concorrentes.

Os níveis de desempenho do Tecon em cada atributo, bem como o desempenho geral do terminal foram atribuídos pelos próprios respondentes. Já á importância de cada atributo foi identificada indiretamente, mediante análise de regressão múltipla, com o auxílio do software SPSS, versão 11.0. A partir da Analise de Regressão, foi obtido um modelo matemático capaz de explicar a relação entre os atributos e a avaliação geral. Finalmente, o modelo foi utilizado para concluir a respeito do Tecon Rio Grande, considerando o contexto competitivo em que está inserido. 


\section{Análise de dados e resultados}

Neste capítulo são apresentadas as características das empresas pertencentes à amostra e os resultados da pesquisa.

\subsection{Qualificação das empresas pertencentes à amostra}

Conforme se pode perceber na Tabela 1, a amostra foi composta principalmente por empresas de médio e grande porte (76,7\% do total).

Tabela 1 - Porte das empresas pertencentes à amostra

\begin{tabular}{c|c|c|c}
\hline Porte das empresas (SEBRAE) & $\mathrm{N}^{\mathrm{o}}$ de trabalhadores & $\mathrm{N}^{\circ}$ de empresas & $\%$ \\
\hline Microempresas & 0 a 19 & 3 & $10,0 \%$ \\
Pequenas & 20 a 99 & 4 & $13,3 \%$ \\
Médias & 100 a 500 & 9 & $30,0 \%$ \\
Grandes & Mais de 500 & 14 & $46,7 \%$ \\
\hline Total & - & 30 & $100,0 \%$ \\
\hline
\end{tabular}

Fonte: Dados da pesquisa

Quanto ao tempo de experiência no comércio exterior, percebe-se que nenhuma empresa amostrada possui experiência de menos de três anos, havendo uma concentração de empresas com experiência superior a 10 anos.

Tabela 2 - Tempo de experiência no comércio exterior

\begin{tabular}{c|c|c}
\hline Experiência no comércio exterior & $\mathrm{N}^{\circ}$ empresas & $\%$ \\
\hline 3 a 5 anos & 2 & $9 \%$ \\
5 a 10 anos & 9 & $39 \%$ \\
Mais de 10 anos & 19 & $52 \%$ \\
\hline Total & 30 & $100,0 \%$ \\
\hline
\end{tabular}

Fonte: Dados da pesquisa

No que diz respeito à atuação, pode-se observar que a maioria das empresas atua tanto na exportação quanto na importação. Isso significa que o nível de serviço foi avaliado como um todo, não avaliando separadamente o nível de serviço em cada tipo de operação.

Tabela 3 - Atuação das empresas

\begin{tabular}{c|c|c}
\hline Atuação no comércio exterior & $\mathrm{N}^{\mathbf{o}}$ de empresas & $\%$ \\
\hline Exportação & 0 & $0 \%$ \\
Importação & 7 & $23 \%$ \\
Exportação e importação & 23 & $77 \%$ \\
\hline Total & 30 & $100,0 \%$ \\
\hline
\end{tabular}

Fonte: Dados da pesquisa

\subsection{Avaliação do nível de serviço do Tecon Rio Grande}

Após sua coleta e tabulação, os dados foram primeiramente analisados por meio de estatística descritiva. Os resultados são apresentados na Tabela 4. 
Tabela 4 - Avaliação do nível de serviço do Tecon Rio Grande por parte das empresas amostradas

\begin{tabular}{c|ccccccc}
\hline Resp. & FRT & CPO & FRM & FRQ & TTP & ATE & GER \\
\hline Média & 3,53 & 2,73 & 2,97 & 1,90 & 2,80 & 3,13 & 2,87 \\
\hline S & 0,8996 & 0,8683 & 0,6687 & 0,8449 & 0,8052 & 0,8604 & 0,6288 \\
\hline
\end{tabular}

Fonte: Dados da pesquisa

A análise da Tabela 4 permite observar que os atributos "custos portuários" (CPO), "fretes marítimos" (FRM) e "tempos de trânsito porta a porta" (TTP), assim como a "avaliação geral" (GER), apresentaram médias ligeiramente inferiores a 3 (igual à concorrência), com desvios padrão variando entre 0,6288 e 0,8683. Já os atributos "fretes terrestres até o porto" (FRT) e "atendimento" (ATE) apresentaram médias ligeiramente superiores a 3 (3,53 e 3,13, respectivamente), com desvios padrão de 0,8996 e 0,8604. Cabe salientar que o atributo "frequencia de navios" (FRQ) foi o que apresentou a menor média $(1,9)$ entre todos os atributos avaliados, tendo sido avaliado o nível de serviço nesse atributo como "pior do que os concorrentes".

A partir dos resultados apresentados na Tabela 4, os dados foram submetidos à análise de regressão múltipla. O modelo obtido, o qual é apresentado na Equação 1, apresentou um valor de $\mathrm{R}^{2}$ de $82,5 \%$, o que significa que 82,5 por cento da variabilidade das respostas foi explicada pelos termos do modelo. Adicionalmente, a Tabela 5 apresenta o teste F para a significância do modelo. O valor encontrado indica uma relação estatisticamente significativa entre as variáveis independentes e a variável dependente GER.

Tabela 5 - Teste de significância para o modelo

\begin{tabular}{c|c|c|c|c|c}
\hline & $\begin{array}{c}\text { Somas } \\
\text { quadradas }\end{array}$ & $\begin{array}{c}\text { Graus de } \\
\text { liberdade }\end{array}$ & $\begin{array}{c}\text { Médias } \\
\text { quadradas }\end{array}$ & F & Significância \\
\hline Regressão & 9,459 & 3 & 3,153 & 40,843 & 0,000 \\
Resíduos & 2,007 & 26 & 0,077 & & \\
Total & 11,467 & 29 & & & \\
\hline
\end{tabular}

Fonte: Resultados da pesquisa.

A multicolinearidade também foi analisada. Hair Jr. et al (2005) afirmam que valores de Variance Inflation Factor (VIF) inferiores a 5 indicam baixa correlação entre as variáveis independentes. A Tabela 6 demonstra que as variáveis do modelo são estatisticamente independentes, não caracterizando multicolinearidade, uma vez que foram encontrados valores de VIF menores do que 1,1. Além disso, a Tabela 6 apresenta os coeficientes da análise de regressão múltipla e a significância estatística dos termos. Observa-se que todos os termos são significativos a um nível de confiança de pelo menos 99,9\% (Sig. $\leq 0,001$ ).

Tabela 6 - Análise de Regressão e estatística de multicolinearidade para o modelo GER

\begin{tabular}{|c|c|c|c|c|c|c|c|}
\hline & \multicolumn{2}{|c|}{$\begin{array}{l}\text { Coeficientes não } \\
\text { padronizados }\end{array}$} & \multirow{2}{*}{$\begin{array}{c}\begin{array}{c}\text { Coeficientes } \\
\text { padronizados }\end{array} \\
\text { Beta }\end{array}$} & \multirow[t]{2}{*}{$\mathrm{T}$} & \multirow[t]{2}{*}{ Sig. } & \multicolumn{2}{|c|}{$\begin{array}{l}\text { Estatística de } \\
\text { colinearidade }\end{array}$} \\
\hline & $\mathrm{B}$ & Desvio padrão & & & & Tolerância & VIF \\
\hline (Constante) & 0,480 & 0,237 & & 2,024 & 0,053 & & \\
\hline $\mathrm{CPO}$ & 0,323 & 0,062 & 0,446 & 5,224 & 0,000 & 0,923 & 1,083 \\
\hline FRQ & 0,417 & 0,063 & 0,560 & 6,672 & 0,000 & 0,955 & 1,048 \\
\hline TTP & 0,254 & 0,066 & 0,325 & 3,858 & 0,001 & 0,946 & 1,057 \\
\hline
\end{tabular}

Fonte: Resultados da pesquisa. 
De acordo com Malhotra (2001), os pesos de cada atributo podem ser calculados a partir da Equação 1, obtida na regressão múltipla:

$$
\text { GER }=0,480+0,323 * \mathrm{CPO}+0,417 * \mathrm{FRQ}+0,254 * \mathrm{TTP}
$$

Para calcular o peso de cada atributo, é preciso obter o valor do "desempenho geral” (GER) quando este se encontra no valor máximo (=5) e os demais se encontram no valor mínimo (=1). A seguir, subtraem-se os valores encontrados com o atributo obtido no máximo e no mínimo. Assim, por exemplo, quando o atributo mais impactante, "frequencia de navios" (FRQ), é avaliado com uma nota 5 e os demais atributos são avaliados com nota 1, a avaliação geral é 3,142. Essa é a maior contribuição possível desse atributo para a avaliação geral. Quando todos os atributos são avaliados com nota 1, a avaliação geral é 1,474. Subtraindo-se 1,474 de 3,142 obtém-se o valor 1,668, que é a contribuição possível devido à frequencia de navios. Repetindo-se o procedimento para os fatores “custos portuários" (CPO) e "tempos de trânsito porta a porta" (TTP), obtém-se os valores de 1,292 e 1,016, respectivamente. Assim, os três atributos somados representam uma contribuição na variação de GER de 3,976. Então, através do modelo, observa-se que, percentualmente, o atributo "frequencia de navios" tem o maior peso (42\%), os "custos portuários" tem peso de $32 \%$ e os "tempos de trânsito porta a porta" tem peso de $26 \%$. A observação da Figura 2 vem reforçar o consenso da literatura revisada, que afirma que, cada vez mais, a qualidade como um todo, ou seja, o nível de serviço é que define a escolha dos clientes. Vê-se que os atributos associados ao tempo (frequencia de navios e tempo de trânsito porta a porta) representam um impacto de $68 \%$ na avaliação geral, enquanto os custos portuários representam 32\%. Além disso, observa-se que a frequencia de navios é o atributo mais importante.

Figura 2 - Impacto dos atributos na avaliação geral

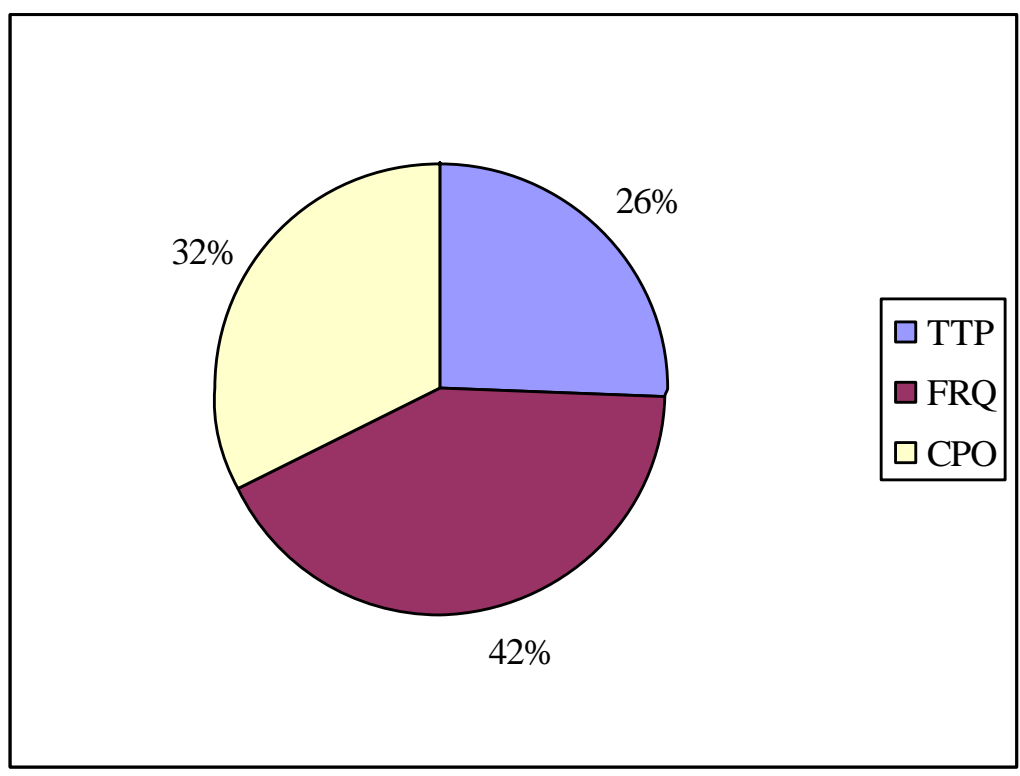

Fonte: Resultados da pesquisa 
A Figura 3 mostra uma análise da sensibilidade da avaliação geral em relação a cada um dos atributos, sendo que as inclinações angulares maiores correspondem aos atributos aos quais a avaliação geral é mais sensível.

Percebe-se que avaliação geral é mais sensível ao atributo "frequencia de navios". A seguir, em ordem de importância, aparecem os atributos "custos portuários" e "tempo de trânsito porta a porta”. Dito de outra forma, modificações nos tempos de trânsito e nos custos portuários levarão mais tempo para alterar significativamente a avaliação geral do que modificações na "frequencia de navios".

Figura 3 - Sensibilidade da avaliação geral em relação aos atributos

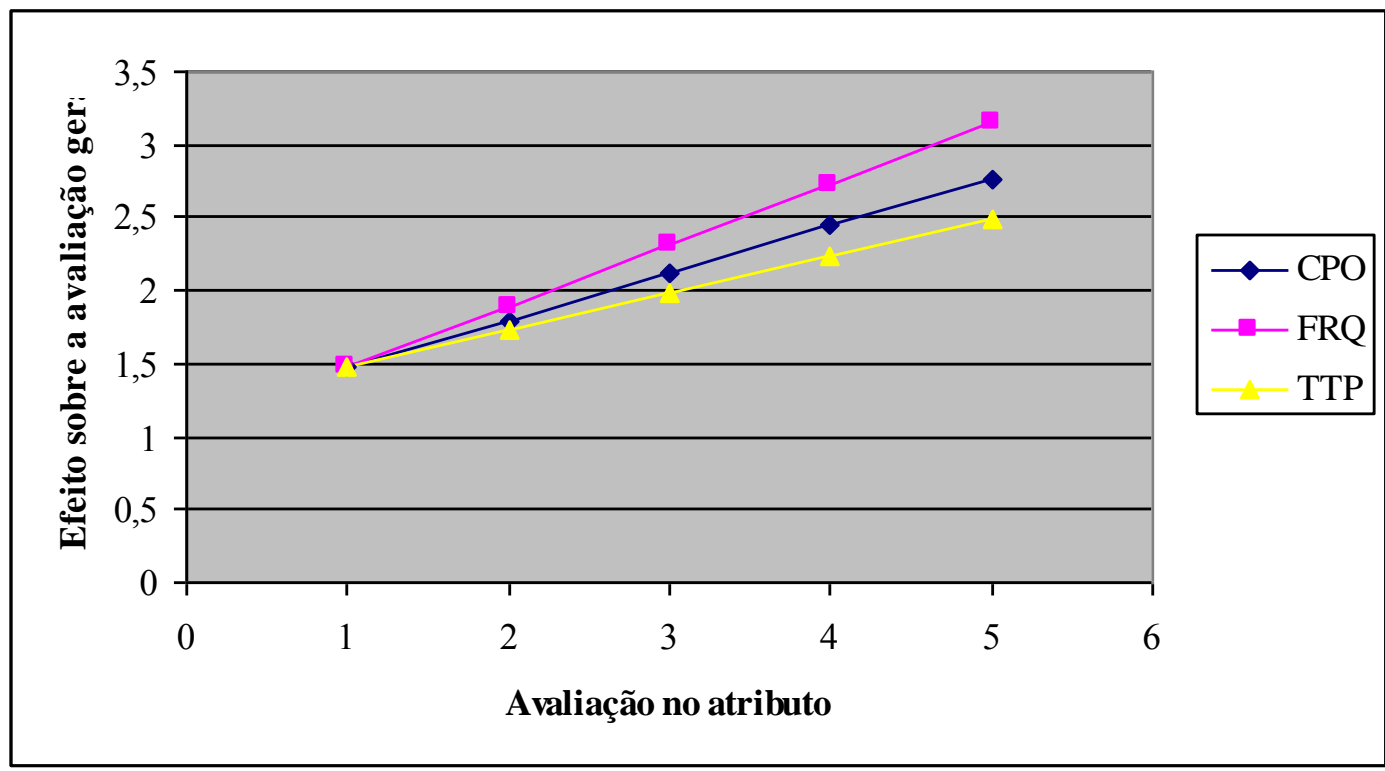

Fonte: Resultados da pesquisa

Esses resultados reforçam que o foi encontrado na literatura da área. Atualmente, os terminais de contêineres tem enfrentado desafios sem precedentes em um ambiente de crescente concorrência e sujeito a pressões cada vez maiores dos diferentes stakeholders (CHANG, LEE, TONGZON, 2008). Entre tais stakeholders, segundo Zan (1999), devem ser considerados três atores principais atuantes no transporte marítimo de contêineres: i) a administração portuária (neste caso, do terminal); ii) os armadores (transportadores marítimos de linha regular) e iii) os usuários (embarcadores e destinatários das cargas). Segundo Vieira e Pasa (2005) e Gomes et al (2010), um porto lida basicamente com dois diferentes tipos de clientes: os armadores (companhias marítimas de linha regular) que escalam no porto e os usuários que o utilizam em suas operações de exportação e importação. A interação entre esses dois tipos de clientes definem o fluxo de mercadorias e influenciam de forma direta a atividade portuária, sendo função da administração do terminal satisfazer e reter esses dois tipos de clientes. Segundo Vieira e Pasa (2005), os usuários tendem a escolher um porto em função dos navios que nele escalam. Em uma linha de argumentação similar, porém mais detalhada, Chang, Lee e Tongzon (2008) argumentam que as 
decisões operacionais de rota e frequencia de navios são tomadas pelos armadores em função de seus custos de navegação, dos custos portuários e de estimativas de demanda nos diferentes portos de origem e de destino, visando maximizar seus resultados. E, a partir das rotas e frequencia de escalas disponíveis, os usuários escolhem os portos de embarque e desembarque mais convenientes, considerando critérios como custos, tempos de trânsito totais, eficiência nas operações e serviços agregados.

\title{
5. Considerações finais
}

A sistemática aplicada mostrou-se capaz de auxiliar, com o uso de métodos quantitativos, a identificação dos principais atributos de serviços que influenciam no desempenho geral do Tecon Rio Grande pela ótica dos exportadores e importadores da serra gaúcha. Os resultados obtidos através do questionário vieram confirmar o que a revisão da literatura indicara: os atributos relativos ao nível de serviço, no atual contexto, tem peso maior do que o atributo custo e a frequencia de navios tem destacada importância. Especificamente, neste estudo, a soma dos pesos dos atributos referentes ao nível de serviço ("tempo de trânsito porta a porta" e "frequencia de navios") representou $68 \%$ do impacto na avaliação geral, enquanto o custo representou os restantes $32 \%$. Percebe-se, portanto, que a retenção dos usuários do porto (clientes exportadores e importadores) é dependente da manutenção (ou aumento) da frequencia de escalas dos navios, ou seja, da retenção dos armadores. E o crescente aumento do poder de barganha dos armadores devido à concentração no setor - alianças globais entre companhias marítimas (CHANG, LEE, TONGZON, 2008) - torna ainda mais crítica essa necessidade.

Reafirma-se, então, a validade e a necessidade de pesquisas aplicadas com vistas a identificações cada vez mais efetivas das reais necessidades dos clientes (tanto usuários como armadores) para a constante melhoria e acompanhamento do nível de serviço logístico-portuário.

\begin{abstract}
The competitiveness of the companies in the international context depends, among other factors, on the improvement of its logistic process as a whole. This process is strongly related to maritime transport, once it represents around $90 \%$ of international shipping cargo. The ports act as links of global supply chain affecting both costs and logistic service level and consequently influencing the competitiveness of exporting and importing sectors that use them. In this context, port service level deserves special attention and it is necessary to evaluate it constantly aiming its improvement. The present paper had as goal identify and evaluate the competitive attributes of Tecon Rio Grande from the Serra Gaúcha exporters and importers view, who are users of Tecon's services. The Tecon's service level in each attribute comparatively with others competitors were evaluated by the respondents and the importance of each attribute were obtained indirectly through multiple regression analysis. The study generated subsidies to Tecon Rio Grande improve its services and to users reassess port choice, once it has identified the importance of the different factors considered by the users in port choice and it has evaluated Tecon's performance in them.
\end{abstract}


Keywords: port service level; serra gaúcha exporters and importers; Tecon Rio Grande.

\section{Referências}

AGÓS, F. E. El Plan Estratégico: un instrumento para la gestión portuaria. Valência: IPEC, 1993.

ARRUDA, J. B. F.; BASTOS, M. M. M. Pesquisa de dados secundários marítimos-portuários: Brasil e Europa. In: XIV ANPET - Anais do Congresso de Pesquisa e Ensino em Transportes, 2000, Gramado/RS.

ATHAWALE, V. M.; MUKHERJEE, P.; CHAKRABORTY, S. Supplier selection using multi-criteria decision-making methods. The IUP Journal of Operations Management, v. 3, n. 3-4, p. 41-60, 2009.

BAKER, G. H. The Carrier Elimination Decision: Implications for Motor Carrier Marketing. Transportation Journal, v. 24, n. 1, p. 20-29, 1984.

BELLO, T. S.; TERUCHKIN, S. U.; GARCIA, A. A. Alterações no perfil das exportações gaúchas. In: CONCEIÇÃO, O.; GRANDO, M.; TERUCHKIN, S. U.; FARIA, L. (Orgs.). Três Decadas da Economia Gaúcha. Porto Alegre:

Fundação de Economia e Estatística (FEE), 2010.

BRASIL. Agência Nacional de Transporte Aquaviário (ANTAQ). Anuário Estatístico Portuário 2009. Disponível em: http://www.antaq.gov.br/Portal/Anuarios/Portuario2009/In-dex.htm.> Acesso em: 29.dez.2011.

BROOKS M. R. Understanding the Ocean Container Market - A Seven Country Study. Maritime Policy and Management, v. 22, n. 1, p.39-49, 1995.

cross ref

BROOKS, M. R. Performance evaluation by North American carriers. Transport Reviews: A Transnational Transdisciplinary Journal, v. 19, n. 3, p. 11-11, 1999.

CHANG, Y. T.; LEE, S. Y.; TONGZON, J. L. Port selection factors by shipping lines: different perspectives between trunk liners and feeder service providers. Marine Policy, v. 32, n. 6, p. 877-885, 2008.

cross ref

DALEY, J. M.; LAMBERT, Z. V. Toward assessing trade-offs by shippers in carrier selection decisions. Journal of Business Logistics, v. 2, n. 1, 1980.

D'ESTE, G. M. Carrier selection in a RO/RO ferry trade Part 2: concept framework for the decision process. Maritime Policy and Management, v. 19, n. 2, p. 127-138, 1992.

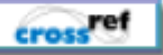

D'ESTE, G. M.; MEYRICK, S. Carrier selection in a RO/RO ferry trade Part 1 . Decision factors and attitudes. Maritime Policy and Management, v. 19, n. 2, p. 115-126, 1992.

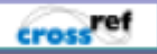

ESTADO DO RIO GRANDE DO SUL. Secretaria de Planejamento, Gestão e Participação Cidadã. Atlas Socioeconômico do Rio Grande do Sul 2010. Disponível em: http://www.scp.rs.gov.br/atlas/default.asp. Acesso em: 30.jan.2012.

FLEURY, P. F. Gestão Estratégica do Transporte. COPPEAD-Universidade Federal do Rio de Janeiro, v.1, 2002, disponível em http://www.cel.coppead.ufrj.br/fr-estrat-trans.htm. Acesso em: 28.ago.2005.

GIL, A. C. Como elaborar projetos de pesquisa. 4. ed. São Paulo: Atlas, 2002.

GOMES, C. F. S.; VIEIRA, G. B. B.; PASA, G. S.; SANTOS, C. H. S. Identificação de hub ports na costa brasileira: comparação entre a análise conjunta e o sad-thor. Revista de la Escuela de Perfeccionamiento en Investigación Operativa, v. 1, p. 1-15, 2010. 
GORDON, I. Marketing de Relacionamento. 3. ed. São Paulo: Futura, 1998.

HAIR JR., J. F., ANDERSON, R. E., TATHAM, R. L.; BLACK, W. C. Análise Multivariada de Dados. Porto Alegre: Bookman, 2005.

KENT, J. L.; PARKER, R. S. International Containership Carrier Selection: Shippers/Carriers Differences. International Journal of Physical Distribution \& Logistics Management, v. 29, n. 6, p. 398-408, 1999.

cross ref

KUMAR, S. A decision support model for the liner shipping competition policy debate. Loeb-Sullivan School- Maine Maritime Academy: Maine, 2003.

LU, C. S.; MARLOW, P. B. Strategic groups in Taiwanese liner shipping. Maritime Policy and Management, v. 26, n. 1 , p. $1-26,1999$.

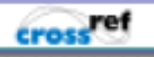

MAGAlA, M.; SAMMONS, A. A New Approach to Port Choice Modelling. Maritime Economics \& Logistics, v. 10, p. 9-34, 2008.

cross ref

MALHOTRA, Naresh. Pesquisa de Marketing: uma orientação aplicada. 3. ed. Porto Alegre: Bookman, 2001.

MILAN, G. S.; VIEIRA, G. B. B. Proposição de um modelo conceitual em torno da prática da governança em cadeias logístico-portuárias. Revista Gestão Industrial, v. 7, n. 4, p. 154-174, 2011.

cross ref

MONTEIRO, A. B. F. C.; MARTINS, W. C.; RODRIGUES, F. H. O processo de decisão do modal no transporte de carga. In: Gestão Logística do Transporte de Cargas. Caixeta-Filho, José Vicente e Martins, Ricardo Silveira (org.). São Paulo: Atlas, 2001.

MUÑOZ, J. M. B. El Puerto de la Bahía de Cádiz: sus áreas de influencia. Cuadernos de Geografía, n. 1, p. 35-54, 1989. Disponível em: <http://rodin.uca.es:8081/xmlui/bitstream/handle/10498/14091/18387330.pdf?sequence=1〉. Acesso em: 15.out.2011.

MURPHY, P. R.; DALEY, J. M. A Comparative Analysis of Port Selection Factors. Transportation Journal, v. 34, n. 1, p.15-21, 1994.

MURPHY, P. R.; DALEY, J. M.; DALEMBERG, D. R. Port Selection Criteria: An Application of a Transportation Research Framework. Logistics and Transportation Review, v. 28, n. 3, p.237-255, 1992.

NOVAES, A. G. N.; VIEIRA, H. F. O nível de serviço logístico-portuário sob a ótica dos exportadores. Gestão e Produção. Florianópolis, v. 3, n. 3, p. 290-306, dez. 1996.

OMC - Organização Mundial do Comércio. Site Institucional. Disponível em: 〈http://www.wto.org>. Acesso em: 05.nov.2011.

PAIXÃO, A. C.; MARLOW, P. B. Fourth generation ports - a question of agility? International Journal of Physical Distribution \& Logistics management, v. 33, n. 4, p. 355-376, 2003.

cross ref

PEYRELONGUE, C. M. El puerto y la vinculación entre lo local y lo global. Revista Eure, v. 25, n. 75, p. 103-120, Santiago de Chile, septiembre 1999.

SAATY, T. L. Método de Análise Hierárquica. São Paulo: Makron Books, 1991.

SAMPIERI, R. H.; COLLADO, C. F.; LUCIO, P. B. Metodologia de Pesquisa. 3. ed. São Paulo: MacGraw-Hill, 2006.

SANTOS, C. H. S.; VIEIRA, G. B. B.; PASA, G. S.; BASSANESI, M. M. R. Exportação nos setores metal-mecânico e automotivo gaúchos: pesquisa de mercado para a seleção de portos. In: Anais do XIV Simpósio de Engenharia de Produção - Simpep. Bauru: Unesp, 2007. 
SOUSA JR., J. N. C. Avaliação da eficiência dos portos utilizando análise envoltória de dados: estudo de caso dos portos da região nordeste do Brasil. Dissertação de Mestrado. Programa de Mestrado em Engenharia de Transportes, Universidade Federal do Ceará, Fortaleza, 2010.

TONGZON, J. L. Portchoice and freight forwarders. Transportation Research Part E: Logistics and Transportation Review, v. 45, n . 1, p. 186-195, 2009.

TONGZON, J. L. Port Choice in a Competitive Environment. IAME 2002 - International Association of Maritime Economists Conference Proceedings. Panama 2002. Disponível em:

<http://www.cepal.org/usi/perfil/iame_papers/proceedings/Tongzon.doc.> Acesso em: 30.01.2012.

TRAN, N. K. Studying port selection on liner routes: An approach from logistics perspective. Research in Transportation Economics, v. 32, n. 1, p. 39-53, 2011.

\section{cross ref}

TUNA, O.; SILAN, M. Freight transportation selection criteria, IAME 2002 - International Association of Maritime Economists Conference Proceedings. Panama 2002. Disponível em:

<http://www.cepal.org/usi/perfil/iame_papers/proceedings/Tuna_et_al.doc.> Acesso em: 30.01.2012.

VALPUESTA, L. L.; MANZANO, J. I. C. Análisis de la actividad económica del puerto de Sevilla y su influencia provincial. Universidad de Sevilla y Autoridad Portuaria de Sevilla. Serie Ciencias Económicas y Empresariales. Número 55. Sevilha: Universidad de Sevilla, 2001.

VIEIRA, G. B. B.; PASA, G. S. Escolha portuária: um estudo piloto para a identificação de hub ports na costa brasileira através de análise conjunta e projetos de experimentos. In: Anais do XXV Encontro Nacional de Engenharia de Produção - Enegep. Porto Alegre, 2005.

VIEIRA, G. B. B.; PASA, G. S.; SANTOS, C. H. S.; BASSANESI, M. M. R.; MACHADO, J. K. O nível de serviço do Tecon Rio Grande a partir da ótica dos usuários. In: Anais do III Simpósio Internacional de Gestão de Negócios em Ambiente Portuário - SINAP. Santos, 2006.

VIEIRA, G. B. B.; RODRIGUES, S. L. A. O Nível de Serviço de um Consolidador de Cargas Marítimas sob a Ótica dos Usuários. Revista Produção On Line, v. 5, n. 2, 2005.

ZAN, Y. Analysis of container port policy by the reaction of an equilibrium shipping market. Maritime Policy \& Management, v. 26, n.4, p. 369-381, 1999.

cross ref

\section{Dados dos autores:}

Nome completo: Guilherme Bergmann Borges Vieira

Filiação institucional: UCS

Endereço completo para correspondência: Av. Venâncio Aires, 134/612, Bairro Cidade Baixa, Porto Alegre-RS, Brasil.

Telefone para contato: (55) 51-99448504.

e-mail:gbvieira@cpovo.net

Nome completo: Roberto Birch Gonçalves

Filiação institucional: UCS

Endereço completo para correspondência: 
Telefone para contato:

e-mail:rbgoncal@ucs.br

Nome completo: Giovana Savitri Pasa

Filiação institucional: UFRGS

Endereço completo para correspondência:

Telefone para contato:

e-mail: giovanapasa@producao.ufrgs.br

Nome completo: Francisco José Kliemann Neto

Filiação institucional: UFRGS

Endereço completo para correspondência:

Telefone para contato:

e-mail:kliemann@producao.ufrgs.br

Enviado em: 14/08/2012

Aprovado em: 18/07/2013 